\title{
Cuadernos de Cultura y la conformación de un ámbito de poéticas comunistas en la Argentina de los años 50
}

\section{Cuadernos de Cultura and the Creation of a Communist Poetry's Area in Argentina in the 1950s}

\section{Resumen}

Este artículo indaga la conformación de un ámbito de poéticas comunistas en el marco de la revista Cuadernos de Cultura en la década de 1950. El programa literario definido desde Cuadernos otorgó un lugar destacado a la difusión y promoción del género poético, a partir de la asidua publicación de reseñas, notas, comentarios críticos y poemas, que incidieron en la conformación de un ámbito específico de "poéticas comunistas", identificadas, de acuerdo a los programas soviéticos que la revista adopta, por su carácter realista y nacional. Se sostiene que ese ámbito reúne a un grupo amplio de poetas, tanto del centro metropolitano como del interior del país, que permite construir un corpus original de "poéticas comunistas", no identificado previamente en el campo de los estudios literarios. En esta dirección, la política "poética" que se pone en juego en la revista redundó en una reconfiguración de los espacios centrales y periféricos del sector de la izquierda comunista del campo poético.

Palabras claves Poéticas comunistas; Cuadernos de Cultura; Zhdanovismo cultural, Realismo; Década de 1950

\begin{abstract}
This paper inquire the configuration of an area of communist poetics in the framework of the magazine Cuadernos de Cultura in the 1950s. It is argued that the literary program defined from Cuadernos gave a prominent place to the diffusion and promotion of the poetic genre, from the assiduous publication of reviews, notes, critical commentaries and poems, that influenced the conformation of a specific field of "communist poetics", identified, according to
\end{abstract}


the Soviet programs that the magazine adopts, for its realism and national character. This field gets together a broad group of poets who come from the metropolitan center and the interior of the country. In this direction, the politic "poetic" that the magazine put into play resulted in a reconfiguration of the central and peripheral spaces of the communist left's sector of the poetic field.

Keywords Communist poetics; Cuadernos de Cultura; Zhdanovism cultural; Realism, The 1950s

\section{Realismo y poesía en la revista Cuadernos de Cultura}

Las políticas culturales y artísticas del comunismo argentino en los años 50 tuvieron a su cargo la tarea de traducir y tratar de adaptar al contexto nacional, signado por la agenda de problemas que imponía el peronismo, las directivas soviéticas codificadas en lo que se conoció, a escala internacional, como "doctrina zhdanovista", por el nombre del encargado de formularla y vigilar su estricto seguimiento: Andréi Zhdanov, el comisario cultural del estalinismo en los tiempos de la posguerra y comienzos de la Guerra Fría. Puntualmente en el caso del arte, esta doctrina implicaba un conjunto de prescripciones en torno a la creación artística, centradas en los lineamientos que, ya desde la década del 30, en la Unión Soviética, conformaban las bases del realismo socialista (Cfr. Slonim, Volkov, Groys). En el contexto de la lucha contra el "imperialismo norteamericano" que sostiene como principal línea de acción política el Partido Comunista (PC) a nivel mundial, las codificaciones culturales de Zhdanov se sustentaron sobre dos cometidos complementarios, que fueron impuestos en la URSS con todo el rigor de un fuerte aparato de control y censura y que, en el resto del mundo se tradujeron, como señala Adriana Petra ("Intelectuales comunistas", Intelectuales y cultura comunista), en una "profesionalización" del trabajo intelectual': por un lado, la

${ }^{1}$ Si hasta los años finales de la Segunda Guerra, en el marco de las políticas frentistas, el resto de los partidos comunistas del mundo no les exigió a sus intelectuales que extendieran su compromiso con las reivindicaciones partidarias al ámbito de la creación artística, la situación se modificó después de 1945, cuando, de acuerdo a Petra, se inicie una búsqueda por "profesionalizar" la participación de los intelectuales ligados al comunismo. Así, a "la concepción clásica que concebía 
lucha contra el "cosmopolitismo", pensado como una derivación a nivel artístico del peligro que el imperialismo significaba para la libertad política y económica de los pueblos; y, por otro, la defensa y el fomento de las culturas y las tradiciones nacionales. ${ }^{2}$ En términos del mismo Zhdanov, se trataba de una tarea doble: "fustigar y atacar con audacia a la cultura burguesa" y "restaurar" e "impulsar" "las más gloriosas tradiciones" ("El frente ideológico" 92-96).

En el marco de la recepción de la doctrina zhdanovista en Argentina, la revista Cuadernos de Cultura, el principal órgano de difusión cultural del Partido Comunista Argentino (PCA) en esos años, -y fundamentalmente, a partir de su séptima entrega, de julio de 1952, cuando Héctor Agosti asume su dirección (Cfr. Tarcus)- constituye una suerte de laboratorio en el que los intelectuales, escritores y poetas comunistas se embarcarán en la tarea -no exenta de tensiones, polémicas y discusiones- de ensayar modos de adaptación de los lineamientos artísticos soviéticos en la construcción de un programa cultural de carácter nacional. Para la formulación de ese programa, los intelectuales comunistas echaron mano en una biblioteca que combinó las fuentes soviéticas con algunas nociones tomadas de los filósofos del pre-romanticismo alemán (la de la poesía como "expresión popular" de Johann Gottfried von Herder, por ejemplo), y, fundamentalmente, con las novedades que aportaba la cultura marxista italiana de posguerra; en particular, con las primeras lecturas de la obra de Antonio Gramsci y de otros críticos y teóricos

la acción común de los intelectuales en función de una intervención puramente política avalada por su capital simbólico" se la reemplazaría por "una concepción profesionalista en la que el intelectual debía comprometer su obra o sus prácticas y competencias profesionales". Esto supuso un cambio tanto en lo que concierne a la tarea y el rol específico que debían cumplir los intelectuales como a sus formas de organización, ahora centradas en la constitución de frentes por especialidad ("Intelectuales comunistas" 109-110).

${ }^{2}$ Para un estudio más exhaustivo del zhdanovismo cultural y su recepción a nivel nacional remito a Petra ("Intelectuales comunistas", Intelectuales y cultura comunista). En sus trabajos, la historiadora indaga las relaciones entre intelectuales y PC en Argentina en el arco cronológico que va desde 1945 hasta 1963 y realiza un exhaustivo análisis del "tono nacional" que la imposición de la doctrina zhdanovista, en el contexto del peronismo, imprimió al discurso comunista. 
literarios como Mario De Micheli o Galvano della Volpe, propiciadas por Héctor Agosti, ${ }^{3}$ y con la recepción crítica del cine y la literatura del neorrealismo italiano. ${ }^{4}$ Si bien, como su nombre lo indica, Cuadernos es una revista políticocultural que excede el campo específico de la literatura para abordar temas que van desde las artes en sus diferentes manifestaciones hasta la filosofía, la historia, la ciencia, la pedagogía o la economía, lo cierto es que, como señala Petra, los debates en torno a la literatura y, podría agregarse, el campo expandido de las artes -las artes plásticas, el cine y el teatro, fundamentalmente- "ocuparon una porción significativamente mayor a cualquier otro campo de interés, tanto en los artículos como en las reseñas bibliográficas" ("Intelectuales comunistas" 143). La literatura y las artes adquieren centralidad y se perfilan como contenidos dominantes en las páginas de la revista. Desde la óptica partidaria, entonces, literatura y arte se constituyen, dentro del espectro amplio de la cultura, como elementos indispensables para deliberar en torno a las posibilidades de un programa cultural comunista, capaz de adaptar a las particularidades del contexto nacional, signado por el fenómeno del peronismo, los lineamientos que emanaban de la doctrina zhdanovista.

En el caso específico de la literatura, podría decirse que tanto el entramado de las polémicas y las discusiones que se suscitan en la revista, como la selección de los materiales que se traducen y difunden y la valoración de los libros reseñados ponen en juego una búsqueda por construir un programa literario que, en la confluencia de este universo de lecturas y fuentes diversas, regule las prácticas

${ }^{3}$ Tal como sostiene Petra en sus estudios citados, ya desde Echeverría (1951) y Para una politica de la cultura (1956) pero, fundamentalmente, en Nación y Cultura (1959) y El mito liberal (1959), Agosti recurrirá a los aportes de Gramsci en función de repensar los problemas nacionales por fuera de la tradición liberal a la que hasta el momento había recurrido de manera privilegiada. Sin embargo, esa apertura de la cultura marxista conocerá un límite preciso: la no discusión de los aspectos fundamentales que constituyen la base del marxismo-leninismo. En efecto, cuando los jóvenes intelectuales comunistas que rodean a Agosti comiencen a utilizar ese novedoso corpus textual para cuestionar las bases doctrinarias soviéticas, se iniciará un proceso de ruptura que culminará con la expulsión de los jóvenes disidentes de las filas partidarias (Tarcus "El corpus marxista").

${ }^{4}$ Petra estudia la recepción del neorrealismo en Argentina. En Alle ("Un boedismo") atiendo particularmente a las discusiones que suscitó en el ámbito cultural comunista la recepción del film de Vittorio De Sica, Ladrones de bicicleta. 
críticas y creativas nacionales. Cuadernos de Cultura se presenta, así, como un espacio de diálogo en el que se ensayan y discuten diversas propuestas, en vistas de la elaboración de un aparato teórico-crítico capaz de orientar tanto las perspectivas de análisis y valoración de la literatura como el mismo trabajo de escritura literaria.

Este programa, en consonancia con las directivas artísticas del estalinismo, se sustentaba en una estética realista basada en la teoría leninista del conocimiento como "reflejo". En estos términos, el realismo suponía que el artista debía no sólo reflejar la realidad en sus obras - una realidad cuya existencia sería independiente de la conciencia-, sino también contribuir a la modificación futura de esa misma realidad, de modo que ese "reflejo" literario constituía en última instancia una herramienta al servicio del cambio social. ${ }^{5}$ En definitiva, el programa literario de la revista -acorde con los presupuestos de la doctrina soviética- se sustentó en un sistema axiológico que identificaba el valor de la literatura con su carácter instrumental, es decir, al servicio del principio pedagógico y moralizante que Máximo Gorki, en su discurso al Primer Congreso de Escritores Soviéticos, de 1934, definía como el deber del escritor de “despertar la responsabilidad colectiva". ${ }^{6}$ Para que lograra cumplir con esa función, esa representación de la realidad, lejos de quedar limitada a la mera copia de la superficie de los fenómenos, debía captar "la realidad en su desarrollo revolucionario"; lo que, en última instancia, terminó por derivar en una discusión en torno al "optimismo" de las obras y, de modo todavía más simplificado, en el problema del “final feliz". Solo a partir

${ }^{5}$ Tal como señala Horacio Crespo ("Poética, política") a propósito de la traducción del artículo de Parsadanov, "La lucha por el realismo en el arte", publicada en el número 15 de la revista, la teoría del reflejo que Lenin desarrolla en Materialismo y empiriocriticismo supone que la operación de conocimiento es la del reflejo del mundo, que existe independientemente de la conciencia del hombre, en la mente humana. Así, dice Crespo, "el sentido del arte y la literatura [...] es que refleje fielmente la realidad objetiva del mundo [...]" a partir de un medio propio, la imagen artística. Dichas imágenes posibilitarían que el artista refleje la realidad y, al mismo tiempo, influya sobre ella a los fines de su transformación (426). Para un estudio crítico de la teoría del reflejo leninista, recomiendo: Lecourt, Sánchez Vázquez.

${ }^{6}$ Dice Gorki: "Los miembros del Partido que actúen dentro del dominio de la literatura deberán ser no solamente maestros de ideología revolucionaria que organizan las energías del proletariado en todos los países del orbe, sino que den muestras de fuerza moral y de verdadera disciplina. Esta fuerza deberá tender, ante todo, a despertar la responsabilidad colectiva" (54). El Congreso de 1934 es el marco en el que la Unión de Escritores Soviéticos, dirigida por el mismo Gorki, define e impone al realismo socialista como único camino estético posible para los escritores soviéticos. 
de la representación del desarrollo revolucionario de la realidad, es decir, del devenir dialéctico que conduciría hacia la sociedad sin clases, lograría una obra literaria aportar un conocimiento profundo de dicha realidad y, por ello, dar un paso hacia la acción transformadora: la praxis, en términos de Lenin. ${ }^{7}$

Sobre la base de esta concepción del realismo, el programa literario de la revista se define en torno a tres ejes. En primer lugar, supone -tal como lo había postulado la doctrina soviética- un enemigo a combatir, el "cosmopolitismo", cuyo horizonte más definido en el ámbito nacional no fueron sólo las expresiones de la renovación literaria sino también los géneros policial y fantástico, el cine norteamericano (en sus vertientes "rosa" y "negra") y las novelas "existencialista" y "psicologista", que la revista asocia a las obras de Jorge L. Borges, Adolfo Bioy Casares, Ernesto Sábato o Manuel Mujica Láinez, en un trazado de confrontaciones que culmina, por extensión, en $S u r .{ }^{8}$ En segundo lugar, ese programa impulsó la búsqueda de una "herencia cultural", a partir de la cual consensuar y legitimar las modalidades de acercamiento de la literatura al mundo popular; "herencia" que progresivamente, siguiendo a Petra, fue delineando una genealogía en tensión con el panteón liberal que el partido había adoptado como propio en los años 30, aunque sin llegar a romper definitivamente con él (Intelectuales y cultura comunista). Por último, en lo que respecta específicamente a las resoluciones artísticas de las obras, el programa literario de la revista puso en el centro del debate el problema en torno al "elemento revolucionario" y a la "comunicabilidad" que debían presentar las obras literarias "populares"; dos cuestiones que remiten al problema del contenido y la forma apropiadas que debían revestir las obras literarias realistas para cumplir con la misión de orientar al lector en su lucha a favor del cambio social.

${ }^{7}$ La definición que quedó establecida en el Estatuto de la Unión de Escritores Soviéticos aprobado en el Congreso del año 1934 estipulaba que "El realismo socialista, método básico de la literatura y de la crítica literaria soviéticas, exige del artista una representación veraz, históricamente concreta de la realidad en su desarrollo revolucionario. Además, la verdad y la integridad histórica de la representación artística debe combinarse con la tarea de transformar ideológicamente y educar al hombre que trabaja dentro del espíritu del socialismo" (Slonim 198).

${ }^{8}$ Jorge Cernadas señala la "moderación parcial" de la crítica de Cuadernos a Sur. No obstante, centrando la mirada en los parámetros valorativos que sostienen el programa literario de la revista, se advierte que los mismos conducían necesariamente a una confrontación con Sur. 
Por un lado, las discusiones en torno al "elemento revolucionario" -relativa al problema de los "contenidos" de las obras y, en última instancia, a la representación realista- conforman un núcleo problemático en el que resuenan los ecos de los principios pautados por la doctrina soviética. En su discurso al Primer Congreso de Escritores Soviéticos, Gorki señalaba que la literatura popular de raigambre materialista, que el realismo socialista recoge como herencia, logra fundir "razón e intuición”, entendiendo por "intuición” la capacidad de vislumbrar en el presente el triunfo final de los oprimidos sobre las clases que los oprimen. Y, gracias a esta conjunción, capta "la acción gestadora de la realidad", es decir, la dialéctica que anuncia el avance de las fuerzas motoras de la historia hacia la revolución proletaria y, después, hacia la sociedad sin clases, motivo por el cual “desconoce por completo el pesimismo" (23). Si la dialéctica materialista enseña que todo momento histórico contiene en sí las contradicciones cuyo desenvolvimiento será superado en una instancia posterior, de síntesis, el "reflejo" artístico tendría como objetivo captar esas contradicciones y mostrar su desenvolvimiento futuro. En diálogo con esta concepción del realismo, podría decirse que tanto de las reseñas como de los artículos críticos y ensayos que se publican en la revista se desprende un sistema de valoración que pone el acento afirmativo en la construcción de tramas narrativas capaces de representar los padecimientos de los trabajadores en las circunstancias de explotación a las que se encuentran sometidos, para derivar, en su desarrollo argumental, en una visión del triunfo que están llamados a cumplir, revistiendo así a la historia narrada de un carácter "ejemplar".

Por otro lado, la cualidad "comunicable", que remite a la forma de las obras, aparece en general asociada a una explícita crítica al simbolismo, la literatura "pura” y a la experimentación vanguardista. Así aparece formulado el problema en la reseña crítica de la antología poética Guatemala (1954), del grupo Poesía Buenos Aires, que escribe Juan Hidalgo para el número 21 de la revista, en donde rescata el interés por intervenir ante los episodios imperialistas sucedidos en Guatemala por parte un grupo que, hasta el momento, se había enfrentado al "problema 
poético, con una postura cerradamente individualista" (120-121). En este sentido, señala que "una poesía [...] con una temática de definida orientación social, no puede perder su comunicabilidad (que es su esencia más entrañable) en los repliegues de un simbolismo más apto para crear ciertos torturados estados de ánimo que los sacrificios de un pueblo en lucha por su libertad" (122). ${ }^{9}$

Ahora bien, si ya el hecho de que el problema en torno a la "comunicabilidad" de las obras se formule en una reseña sobre una antología poética adelanta el rol que la poesía va a cumplir en ese programa literario de la revista, es importante señalar, además, que una de las más claras y concisas definiciones del realismo literario tal como lo postula la revista aparece en un artículo de Juan José Manauta, publicado en el número 8, de octubre de 1952, dedicado a la poesía de Pablo Neruda. Allí, Manauta puntualiza: "El marxismo enseña que ningún conocimiento de la realidad es autosuficiente, sino necesario para cambiar la propia realidad, y el arte, como forma peculiar del conocimiento de la realidad, contribuye a ese cambio" (“"Canto General"” 48). Me interesa destacar, con énfasis, el hecho de que esta definición tan didáctica del realismo de acuerdo a la teoría leninista del reflejo pertenezca nada menos que al comentario crítico que un poeta -si bien la obra de Manauta es, en términos cuantitativos, mayormente narrativa, su inicio en la literatura se da desde la poesía- ${ }^{10}$ realiza de la obra de otro poeta -“el” poeta comunista latinoamericano por antonomasia-, pues hasta ahora los estudios sobre realismo socialista han quedado mayormente circunscritos a los problemas de la narrativa. Si bien ya en 1945, en su Defensa del realismo, Agosti había incluido tres breves ensayos sobre poesía - uno dedicado a discutir la idea de la existencia de una "nueva generación” de poetas, sugerida en el primer número de la revista Canto, otro a Tuñón y otro a Juan L. Ortiz-, lo que demuestra que la asociación entre realismo y poesía no es nueva, lo cierto es que una década más tarde, y especialmente desde la revista Cuadernos, la poesía adquiere en el marco

${ }^{9}$ En Alle ("Un boedismo") estudio el modo en que estos tres aspectos se abordan en el caso de la narrativa a partir del concurso de cuentos que organiza la revista en 1954.

${ }^{10}$ Efectivamente, su primer libro es de poesía y, además, como veremos más adelante, va a publicar en el mismo espacio de la revista su poemario Entre dos ríos. 
de los programas partidarios una visibilidad mayor y se constituye como una de las vertientes de reflexión más productivas en torno al problema del realismo, un aspecto que no ha sido explorado por la bibliografía historiográfica y crítica previa. Asimismo, la aspiración a hacer de la poesía "el instrumento de la fraternidad viril" (105) que Agosti pedía a los poetas, ahora se trocará en el requerimiento puntual y específico de filiación partidaria. En otras palabras, la adscripción al comunismo por parte de los poetas, en tanto instrumento de conocimiento de la realidad, se volverá una condición indispensable para la configuración realista de las obras. Así, en su artículo sobre Neruda, Manauta descubre en el poeta chileno una "preocupación consciente [...] por arraigar [...] su poesía en la realidad"; preocupación que liga sus búsquedas poéticas a una estética de cuño realista. Entendido, tal como él mismo acaba de afirmar, como un modo de conocimiento de la realidad que permite operar sobre ella para transformarla, la filiación comunista de Neruda habría contribuido, asegura Manauta, a una "compenetración más estrecha con la realidad de su país y de América", es decir, al "conocimiento de los hombres, de los lugares de trabajo, formas de explotación, sufrimientos, luchas y triunfos del hombre y de la sociedad por el progreso, la libertad y la justicia" (48). Comunismo, realismo y poesía aparecen, aquí, según vemos, vinculados de un modo indisociable.

Pero, además, esta estrecha ligazón entre poesía y realismo, lejos de ser circunstancial o limitada a este artículo en particular, ilumina la importancia y la centralidad que los programas literarios del comunismo argentino en los años 50 otorgaron al género poético, lo que propicia una reconsideración de varias ideas críticas e historiográficas cristalizadas en torno al realismo socialista, que, como señalé, tienden a limitar sus alcances a la narrativa y, en especial, a la novela. Por el contrario, tal como buscaré demostrar en este trabajo, el programa realista definido en la revista tuvo en la poesía un ámbito de despliegue de gran productividad y desarrollo, que tendió a generar mayor nivel de acuerdo entre los escritores y, por ende, suscitó menor grado de conflictividad y de polémica a la hora de definir y trazar los lineamientos para la crítica y la creación poética. 
En efecto, basta ojear rápidamente los 44 números de Cuadernos que se publican en la década del 50 para corroborar el interés que la revista sostiene por el género poético, en el marco más amplio del lugar central otorgado al debate sobre la literatura y el arte. Me interesa, a los fines de iluminar ese interés por la poesía, detallar algunas de las notas, selecciones de poemas, traducciones y artículos críticos sobre poesía que se publican en sus páginas: en el número 6 (de mayo del 52), poemas de Nazim Hikmet, traducidos por Raúl González Tuñón y Amaro Villanueva; en el 7 (de julio del 52), el primero bajo la dirección de Agosti, un artículo de René Lacorte sobre la poesía de Víctor Hugo, ante el 150 aniversario de su nacimiento, y en la sección "De cada cual lo suyo" una nota titulada "El mundo de la poesía", en el que se relata la importancia y la atención que la Unión Soviética brinda a los poetas; en el número 8 (de octubre del 52), además del artículo de Manauta sobre Neruda al que recién me referí, aparecen poemas de Nikola Vapzarov, traducidos del inglés por Julio Galer; en el 9/10 (de febrero de 1953), una traducción de un ensayo de Paul Eluard, "La poesía de circunstancias"; en el número 11 (de abril del 53), un poema de Tuñón dedicado a la muerte de Stalin; en el 13 (de octubre del 53), unos poemas de Paul Eluard traducidos por Héctor Yánover y en "De cada cual lo suyo" una nota titulada "La herencia de Maiakovski" dedicada a los debates suscitados en la URSS en torno a la obra del poeta futurista; en el 14 (de enero de 1954), se reproduce en la sección “Crónicas del tiempo" el discurso leído por José Pedroni en Esperanza, en un homenaje en conmemoración a los 30 años de la publicación de su primer libro de poemas, La gota de agua; en el 15 (de marzo del 54), como homenaje tras recibir el premio "Stalin" en la URSS, un ensayo de Neruda titulado "Hablando en la calle" y también un poema de su autoría; en el número 17 (de agosto del 54), un ensayo de Tuñón sobre Neruda, como introducción a la publicación de su poema "A las Américas", y otro ensayo de Amaro Villanueva sobre la poesía de Pedroni; en el 18 (de octubre del 54), un poema de Tuñón, "Alguien viene cantando", que celebra la llegada a la Argentina de Ilya Erhenburg, y en "Crónica del tiempo", las noticias en torno a los sucesos de "La semana nerudiana en Santiago de Chile"; en el 23 (de diciembre del 55), un 
poema de Pedroni dedicado a la desaparición del médico rosarino, Juan Ingalinella; en el 24 (de marzo del 56), una traducción de "Canto del hacha" de Walt Whitman en el marco del "año de homenaje" al poeta, instituido por el Consejo Mundial de Partidarios de la Paz en conmemoración al centenario de Hojas de hierba; en el 27 (de septiembre del 56), se publica el poemario de Manauta, "Entre dos ríos"; en el 29 (de mayo de 1957), un anticipo del poemario que Tuñón publicará a finales de ese mismo año, A la sombra de los barrios amados, con una nota introductoria firmada por las iniciales J.C.P (fácilmente atribuibles a Juan Carlos Portantiero); en el 31 (de septiembre del 57), un llamamiento a los escritores a la lucha por la paz, titulado "Carta a los escritores", de Neruda; en el número 34 (de marzo del 58), un artículo de Tuñón, "Maiacovski de nuevo entre nosotros" a propósito de la publicación de las Obras escogidas (Editorial Platina, 1957) del poeta soviético, cuya selección, traducción y comentarios estuvieron a cargo de la traductora y también poeta, Lila Guerrero; en el 41, de junio del 59, Floreal Mazia escribe una crónica sobre Antonio Machado. Y ya afuera del período recortado, pero, como veremos sobre el final de este trabajo, de enorme incidencia para pensar el espacio de la poesía en el ámbito cultural del PC, hay que destacar la publicación, en el número 49 (de septiembre-octubre de 1960), de las discusiones que tuvieron lugar en la reunión de "poetas comunistas" convocada el 16 de julio de ese año por la Comisión de Asuntos Culturales del PCA, bajo el título "Un debate sobre poesía".

La enumeración no contempla las reseñas de libros de poesía, cuyo número es también considerable si se tiene en cuenta que, aun cuando la literatura haya ocupado un lugar central, se trata de una revista cultural en un sentido más amplio. Cito solo algunas, tratando de focalizar en las reseñas de poetas que fueron activos colaboradores de la revista o cuyos nombres, tanto como el de los encargados de escribirlas, circularon con asiduidad en sus páginas. En el 13, Yánover reseña la edición de Poemas de Hikmet publicada por Lautaro y en el 18, la de los Poemas de Vapzarov, de la misma editorial; en el 19 (de diciembre del 54), Bernardo Edelman escribe sobre Todos los hombres del mundo son hermanos, de Tuñón, un relato de viaje por los países socialistas que contiene numerosa cantidad de poemas; 
en el 23 (de diciembre del 55), Margarita Aguirre realiza una crítica un tanto severa de Poemas con habitantes de José Portogalo, a la que el mismo autor responde en el número siguiente (de marzo del 56), en la sección "Polémicas", sin repercusiones posteriores; Manauta reseña Despiertan las fogatas, de Elvio Romero, en el 21 (de mayo del 55) y, en ese mismo número, Juan Hidalgo escribe la de la antología Guatemala, del grupo Poesía Buenos Aires; en el 28 (de marzo del 57), Manauta escribe sobre El alma y las colinas de Juan L. Ortiz y sobre Cantos humanos de Mario De Lellis; en el 29 (de mayo del 57) Nélida Etcheverry, sobre Violín y otras cuestiones, el primer libro de Juan Gelman; en el número 30 (de julio del 57), Gerardo Pisarello publica su reseña de Monsieur Jaquin, de Pedroni; en el 34, Portogalo escribe la de A la sombra de los barrios amados de Tuñón; en el 36 (de junio del 58), Manauta, la de Libro de homenaje, del jujeño Jorge Calvetti, y Marcelo Ravoni, la de Bandoneón de papel, el primer libro de poemas de Héctor Negro; en el 37 (de septiembre del 58), Manauta, la de Cancionero y romancero de ausencias, una edición póstula de Miguel Hernández; en el 41, Fina Warschaver reseña Poetas chinos, traducido del francés por Álvaro Yunque, y Ernesto Guelperin, El tiempo es un barrio de Julio César Silvain; en el 43 (de septiembre/octubre de 1959) Gerardo Pisarello escribe sobre tres poemarios de reciente aparición: La pampa y yo, de Ana María Lasalle, Los muelles insumisos, de Rosario Mase y Poemas con bastón, de Arnoldo Liberman.

Este recuento de notas y reseñas ofrece un muestreo ciertamente locuaz del lugar de relativa importancia que ocupa la poesía en las páginas de la revista en el espectro de su preocupación por la literatura y el arte. En efecto, de estos datos podemos inferir que la poesía constituía un ámbito de interés primordial al momento de definir un programa literario realista y nacional. Podría destacarse, además, la mirada internacional del campo de la poesía, que atiende a nombres y obras de alta valoración en el PC a nivel mundial -desde Hikmet a Maiakovski, ${ }^{11}$

${ }^{11}$ Después de su suicidio, la figura de Vladimir Maiakovski fue cubierta con un manto de silencio en las filas partidarias. Esta situación cambia hacia 1935, cuando Stalin lo "consagra" como el "gran poeta de la revolución". En efecto, en diciembre de 1935, cinco años después del suicidio del poeta, Stalin publica en el diario Pravda, el órgano periodístico oficial del PCUS, una nota en 
pasando por Neruda, Eluard, Hernández-, y la recurrencia con que se publican poemas de ocasión, para celebrar, conmemorar, lamentar o denunciar acontecimientos de impacto actual o se adelantan traducciones de poemas de algunos autores que, en los años sucesivos, serán publicados por las editoriales del PC, como Hikmet o Vapzarov, en lo que podría verse también una suerte de estrategia de mercado. Pero, además -y fundamentalmente-, la persistencia de ciertos nombres de poetas argentinos, tanto en calidad de escritores como de objeto de la escritura -Pedroni, Manauta, Villanueva, Tuñón, Portogalo- constituye una clave importante para pensar las tramas de relaciones que se tejieron entre estos poetas en el mismo espacio de la revista -de hecho, son ellos mismos quienes escriben mutuamente las reseñas y artículos críticos de sus obras-y su proyección en la conformación de un ámbito específico de "poéticas comunistas" que no ha sido objeto de atención de la crítica literaria especializada. Podría decirse, entonces, que la revista pone en funcionamiento una política "poética" que permite dilucidar tanto las diversas estrategias de promoción, difusión y circulación de la poesía en el entorno partidario como, al mismo tiempo, las redes de sociabilidad que vinculan a los poetas comunistas entre sí.

De esta lista de nombres propios de poetas que dinamizan la revista y cuyas producciones son difundidas en sus páginas se desprenden, asimismo, dos datos más. El primero, de fundamental interés en función de reflexionar en torno a la índole "nacional" de la cultura que sostenía como cometido principal la doctrina zhdanovista adoptada por la revista, tiene que ver con la confluencia de poetas de provincias -Pedroni en primer lugar, Manauta y Villanueva, después, y en menor medida, pero igualmente presente, Juan L. Ortiz- y de poetas sumamente identificados con la ciudad de Buenos Aires como Tuñón y Portogalo, que

la que señala que "Maiakovski ha sido y continúa siendo el mejor poeta, el más talentoso, de nuestra época soviética. La indiferencia a su memoria y con respecto a sus obras es un crimen" (Lenin y Stalin 113). De acuerdo a Solomon Volkov, la exaltación que hace Stalin de la figura del poeta vanguardista es un gesto pragmático que se explica en el contexto de ese año en el que "estaba preparando el terreno para destruir a sus adversarios políticos" y, más allá de la política del terror que finalmente impondrá, "también quería ganarse a algunos para su causa [...] Y muchos de ellos adoraban la poesía de Mayakovski" (129). 
conforman un corpus original de "poéticas comunistas" hasta ahora no identificado por la crítica literaria. De acuerdo a la hipótesis que sostengo en este trabajo la amplitud territorial de estas poéticas comunistas, tal como estas se presentan en la revista, indica que, en última instancia, la posibilidad de pensar en una tradición poética "nacional" termina por incidir en la apertura del campo literario desde el centro metropolitano hacia los espacios provinciales. ${ }^{12} \mathrm{El}$ segundo dato que arrojan estos materiales sobre poesía que se publican en la revista es la incorporación hacia mediados de la década de una nueva generación de poetas, la mayoría de ellos nucleados en el grupo de poesía "El Pan Duro", que en los 60 romperá con el PCA. En efecto, Gelman, Yánover, Masse, Silvain, Negro, Bignozzi se suman a los nombres recurrentes de poetas provinciales y porteños que dominan el ámbito de poesía de la revista en la primera mitad de la década. En esta dirección, la revista es también un escenario privilegiado para indagar la emergencia, hacia 1955, de una serie de ideas y valoraciones alternativas acerca de las prácticas críticas y creativas que culminará, en los primeros años de la década siguiente, conformando un programa en abierta ruptura con los lineamientos hegemónicos.

En las próximas páginas revisaré sucintamente la reunión de poetas de provincias y porteños que propicia la revista Cuadernos de Cultura en función de la conformación de un ámbito específico de poéticas comunistas identificadas por su tendencia nacional. En segundo lugar, y ya desde una perspectiva eminentemente literaria, iniciaré un análisis -que obviamente tendrá que ser continuado en futuros estudios- de los términos críticos que permiten agrupar, bajo el paraguas del realismo, producciones poéticas tan disímiles como las de los poetas que circulan por las páginas de la revista. Por último, a modo de cierre, me situaré hacia el comienzo de la década del 60 para pensar las tensiones entre estas poéticas

\footnotetext{
${ }^{12}$ Para pensar esta relación entre espacios centrales y periféricos del campo parto de los desarrollos teóricos de Ana Teresa Martínez ("Intelectuales de provincia") acerca de los vínculos, las tensiones y las redes que se tejen entre las diversas escalas del campo intelectual. Asimismo, se toman en cuenta los estudios de Pierre Bourdieu acerca de la dinámica de funcionamiento de los campos literario, intelectual y político ("Las reglas del arte") y las recientes investigaciones que, en línea con las de Bourdieu, lleva a cabo Gisèle Sapiro para analizar las estrategias y las tomas de posición involucradas en el proceso de recepción de la doctrina zhdanovista y su incidencia en la conformación de un ámbito de poéticas comunistas, en el marco de la revista.
} 
comunistas de los años 50 y aquellas otras que, en la década siguiente, renovarán las búsquedas artísticas de la poesía, aunque esa renovación los lleve, apenas unos años después, a romper con el PCA.

\section{Del "descontento campesino" a la poesía "comunista". Cuadernos de Cultura y la conformación de un ámbito nacional de poéticas comunistas}

En 1943, en su antología Poetas sociales de la Argentina, Álvaro Yunque ubicaba al poeta santafesino José Pedroni y al entrerriano Amaro Villanueva -junto con otros poetas de provincias como Luis Gudiño Kramer y Ema Barrandeguy, también entrerrianos- bajo la rúbrica de "poetas del descontento campesino". En ese "libro panorámico", tal como él mismo lo designa, Yunque se proponía visibilizar más de un siglo de poesía social argentina, que, según denunciaba, había sido sistemáticamente negada y ocultada por los "ideólogos" de las clases dominantes (Tomo I, 9). Para ello, seleccionaba y clasificaba esos materiales que, según su perspectiva, habían sido descartados del canon oficial, en un arco que va desde los "poetas anónimos y gauchescos" hasta el escalón más alto de la evolución, el de los "poetas comunistas". Si hay un rasgo, de acuerdo al autor, que caracterizaría a los poetas del descontento campesino, a los que ubica dos peldaños antes que a los comunistas (en el medio sitúa a los “poetas judíos") ${ }^{13}$, sería su afán por dar voz a "la pauperización, el analfabetismo, la miseria orgánica" que padece el hombre de campo. Así, mientras los "ideólogos caros a los latifundistas" se lamentan de la pérdida de "arcádica pureza" y "candidez eglógica" del campo, estos poetas vendrían a mostrar, por contraste, que lo que el campesinado está perdiendo progresivamente no es ninguna clase de bucólica esencia sino su "idiotismo", término que Yunque recupera de Marx (Tomo II, 7). Los poetas comunistas, por su

\footnotetext{
${ }^{13}$ Yunque argumenta esta categoría del siguiente modo: "El poeta impugnador del antisemitismo como lo sería el del antiindianismo [sic] del antinegrismo o del antigringuismo es un poeta social. En él se recoge, para el historiador del futuro, una manera álgida de la lucha de clases que caracteriza el paso de la sociedad burguesa hacia una sociedad de superior estructura" (Tomo II, 117).
} 
parte, serían los que representan más cabalmente en la actualidad la poesía social en Argentina. Entre ellos, Yunque ubica a Portogalo, Tuñón, Juan L. Ortiz, Alfredo Varela -que en los años 50 se destacará fundamentalmente por su novela El río oscuro, publicada en 1943 y llevada al cine por Hugo del Carril en 1952, con el título de Las aguas bajan turbias-, Lila Guerrero -reconocida en Cuadernos principalmente por su labor como traductora- y otros que no participarán de la revista como Cayetano Córdova Iturburu -desvinculado del PCA en 1948 a raíz de una fuerte polémica con la dirigencia partidaria en torno a la importancia de las vanguardias $-{ }^{14}$, Horacio Raúl Klapenbach, Eloísa Ferraría Acosta, Clara Rafael y Félix Marthoz.

Ahora bien, la destacada visibilidad que, en la década siguiente, cobran tanto Pedroni como Villanueva en la revista Cuadernos de Cultura (y también Gudiño Kramer, aunque en su caso inclinado hacia el ámbito de la narrativa, ${ }^{15}$ y Manauta, quien publicaría su primer libro de poemas, La mujer de silencio, un año después de la antología de Yunque) parece indicar un rápido desplazamiento ascendente de estos poetas de provincias desde el lugar del "descontento

${ }^{14}$ En 1948, el partido convoca a una reunión plenaria de intelectuales comunistas que será el inicio de una polémica entre Rodolfo Ghioldi y Cayetano Córdova Iturburu, cuyo final estará signado por la expulsión del segundo de las filas partidarias. En esa reunión se enfrentaron, tal como muestran Ana Longoni y Horacio Tarcus, dos posiciones: por un lado, la oficial, "sostenida en primer término por Rodolfo Ghioldi", que promovía "la necesidad de adoptar un canon estético realista único para la creación cultural de los artistas comunistas", y, por otro, la representada por Córdova Iturburu, "de mayor apertura a la vanguardia y a la libertad en la creación". Según señalan los autores, la posición sostenida por Iturburu era compartida por un buen número de artistas y escritores que, al igual que éste, "se resistió a la embestida que acusaba a las formas modernistas de propagar "el irracionalismo, el antihumanismo, la reacción"” (55). Las discusiones que se produjeron entre ambos en esa reunión continuaron luego por correspondencia privada. Mientras Ghioldi, en esas cartas cruzadas, sostenía la necesidad, y la obligación, de rechazar cualquier influencia del modernismo en el arte, Córdova Iturburu, por su parte, afirmaba que "no es posible un arte revolucionario, nuestro, comunista, sin la utilización de los elementos estéticos y técnicos proporcionados por la gran experiencia artística y literaria de nuestra época" (57). Para más detalle de la polémica y la expulsión de Iturburu remito al artículo de Ana Longoni y Horacio Tarcus y a los trabajos ya citado de Petra.

${ }^{15}$ En efecto, Gudiño Kramer integrará, junto con Raúl González Tuñón, Raúl Larra y Gerardo Pisarello, el jurado del concurso de cuentos que organiza la revista y cuyos relatos ganadores se publican en el número 19, de diciembre de 1954. También Héctor Agosti estaba anunciado como parte del jurado, pero finalmente no pudo tomar parte de las decisiones porque se encontraba detenido desde el 14 de julio de ese año en la cárcel de Villa Devoto, de donde saldrá recién luego del derrocamiento de Perón. 
campesino" hacia el de "comunistas". En este sentido, podría decirse que los programas culturales que el PCA define y promueve en la década del 50 propiciaron modificaciones en las clasificaciones delimitadas por Yunque en la década anterior, al punto de que el "descontento campesino" o, en términos más generales, los "problemas" del pueblo argentino -desde el campo a la ciudad-, considerados desde una amplia gama de perspectivas y enfoques, se vuelven un problema poético comunista de máximo interés.

Ese ámbito de poéticas que se conforma en la revista Cuadernos reunió a un grupo amplio de poetas, ya reconocidos en el campo cultural y literario, en sus diferentes escalas, que provienen tanto del centro metropolitano -Portogalo, Tunón, también Lila Guerrero, cuya presencia en la revista es más bien lateral y limitada a su labor de traductora-, como del interior del país -Villanueva, Manauta, Pedroni, Juan L. Ortiz-.${ }^{16}$ En este sentido, se puede afirmar que la conformación de ese ámbito de poéticas redundó en una reconfiguración de los espacios centrales y periféricos del sector de la izquierda comunista del campo específico de la poesía, que impulsó hacia un lugar central a los poetas de provincias, cuya poesía establecía algún tipo de afinidad con esos espacios de pertenencia. Por otro lado, implicó una reorientación en las búsquedas de los poetas porteños, quienes comenzaron a explorar diversos modos de acercamiento a las temáticas nacionales o intensificaron los caminos ya recorridos en esta dirección. ${ }^{17}$

En el marco de ese programa literario que propiciaba la construcción de una herencia nacional comunista, de índole popular, capaz de "expresar" la realidad del pueblo argentino y movilizarlo hacia la lucha (de eso se trata, en última instancia, esa misión de transformar la realidad que se adjudica a la literatura

\footnotetext{
${ }^{16}$ Hay que destacar que a Juan L. ya Yunque lo había clasificado como "poeta comunista" en su antología y Héctor Agosti le había dedicado un muy elogioso ensayo incluido en Defensa del realismo. Para un estudio de la poesía social de Juan L. y sus vínculos con el PC remito a la lectura de Agustín Alzari ("La poesía social de Jual L. Ortiz").

${ }^{17}$ Así, por ejemplo, en 1945 Tunón había publicado Primer canto argentino, un poemario que, pensado como una intervención ante las elecciones del 24 de febrero de 1946, recorre diversos tópicos asociados a la Argentina, desde el canto a los próceres y la exaltación de los símbolos nacionales hasta la productividad de las tierras y el trabajo. Sin embargo, la mirada nacional de ese poemario está asociada aún al relato del liberalismo del que, en los años 50, el PCA comienza a desligarse.
} 
realista), la posibilidad de expandir el foco de interés en torno a la poesía desde los poetas del centro metropolitano hasta los de provincias, cuya poesía establecía algún tipo de afinidad con esos espacios de pertenencia, se ofrecía como una opción ciertamente eficaz a los fines de "nacionalizar" el sistema poético comunista. En última instancia, el emplazamiento en pie de igualdad de este conjunto de poéticas territorial y estéticamente distanciadas, tal como se advierte en las decisiones editoriales de la revista Cuadernos, rearma las redes de relaciones entre centro y periferia de ese sistema, un aspecto de los programas partidarios que no ha sido trabajado previamente. Para decirlo a partir de las clasificaciones de Yunque, que de algún modo fijan un estado de situación de la poesía comunista en los años del antifascismo, los poetas del "descontento campesino" se desplazan ahora hacia la categoría más alta de la poesía social, la de "poetas comunistas".

Esta preocupación por extender el espacio de la literatura comunista -y en términos más generales, la cultura comunista- desde el centro metropolitano hacia las escalas provinciales, regionales y locales del campo es explícita en la revista y, de hecho, atraviesa gran parte de las reflexiones de los escritores y poetas en torno al problema de la literatura nacional. Asimismo constituye un aspecto todavía no explorado de la revista. En esta dirección, en una reseña para el número 29, de mayo de 1957, sobre el libro de cuentos titulado Pan curuica, del correntino Gerardo Pisarello, Raúl Larra, un escritor de activa participación en la revista -como el mismo Pisarello-, reflexiona sobre las posibilidades de desarrollo de una literatura regional "como condición necesaria para la estructuración de una gran literatura nacional" (109). De acuerdo a Larra, para ese desarrollo sería necesario que el escritor provinciano "no se desarraigue", lo que no implicaría que tenga necesariamente que fijar su residencia en su lugar de origen. Por el contrario, se trataría de un arraigo "espiritual" y no meramente "geográfico":

[...] importa que el escritor no se desarraigue, que mantenga sus vínculos, sus experiencias más profundas, sus vivencias del entorno nativo. Nuestra literatura necesita que los escritores de tierra adentro permanezcan fieles 
en espíritu al paisaje que los acunó y formó. Porque esa fidelidad es el camino más seguro para que se engrandezca la literatura nacional. La confluencia de todas las voces provincianas en un registro común es la garantía de esa grandeza. Y este nuevo libro de Pisarello se suma a los de aquellos que como Villanueva, Ortiz, Gudiño Kramer, Manauta y otros trabajan para rescatar el rostro total de la patria. (110)

Dos ideas pueden derivarse de la cita. En primer lugar, se destaca el hecho de que Larra señale, entre los escritores que trabajan en el camino de "rescatar el rostro total de la patria", a varios de los poetas provinciales que la revista promociona y difunde en sus páginas de manera sobresaliente; lo que refuerza la afirmación acerca de la importancia de la poesía en el marco más amplio del programa literario comunista de los años 50. En segundo lugar, la cita ilumina la centralidad que ese programa otorga a la dimensión provincial del campo literario como instancia fundamental para pensar en una literatura nacional -"la confluencia de todas las voces provincianas en un registro común"-, tanto en lo que respecta al alcance geográfico como a la representación literaria realista, lo que Larra define como la "fidelidad" al "entorno nativo".

En este punto, podría decirse que, en el marco de la recepción del zhdanovismo y de la confrontación con el "sistema de representaciones sobre lo nacional" puesto en marcha por el peronismo (Petra "Intelectuales comunistas" 240), la revista da un paso más sobre algunos de los incipientes planteos sobre la “argentinidad" de la poesía que Agosti había formulado en la década anterior. Lo que la revista hace, así, es desplegar las potencialidades poéticas críticas y creativas de ese "aliento geográfico argentino", de ese "signo de la argentinidad - de la argentinidad que es posesión cósmica de la tierra y no versitos con guitarras y cintas celestes y blancas-" (103) que Agosti reclamaba en Defensa del realismo a la poesía nacional de la década anterior. Ese cometido que la generación del 40, para Agosti, no había podido cumplir por estar gran parte de ella sumida en la "melancolía o, mejor, si se quiere, la angustia a la soledad" que era la consecuencia 
directa de hacer nacido y crecido en medio de guerras y matanzas "devastadoras" (103), lo vienen a concretar ahora, sobre un terreno que las políticas culturales sostenidas por el partido volvían más firme y propicio, los poetas comunistas nucleados en torno a Cuadernos.

En este sentido, estas reconfiguraciones del campo de la poesía comunista se fundamentan en -y al mismo tiempo favorecen- una reorientación en los parámetros valorativos en torno de los cuales se lee, se critica y se proyecta la escritura de poesía. En la búsqueda de la "confluencia de todas las voces provincianas en un registro común" planteada por Larra, los análisis de poesía que propicia la revista atienden fundamentalmente a los diversos modos de acercamiento a temáticas, lenguajes, expresiones culturales, que podrían definirse, desde los programas del partido, como "nacionales"; concretamente, como característicos y distintivos del pueblo argentino, es decir, de raigambre popular, sin caer en el costumbrismo de los "versitos con guitarra y cintas celestes y blancas" que Agosti criticaba. De acuerdo a lo que estipulaban los programas partidarios, la conformación de ese ámbito poético en la revista responde al propósito de construir una tradición poética nacional, ligada al pueblo, que cada poeta recuperaría y, al mismo tiempo, ayudaría a consolidar. De hecho, este tópico aparece casi de modo unánime en el conjunto de las notas, comentarios críticos, ensayos y reseñas de poesía. A partir de la definición de la labor poética que realiza Portogalo en su respuesta a la crítica de Margarita Aguirre, podría decirse que el aparato teóricocrítico sobre poesía que se pone en funcionamiento en la revista parte de la idea central de que el poeta "sale del pueblo para 'dirigirse' a él como pueblo [...] con su expresión busca ser fiel a su clase, consustanciado con lo heredado de una cultura nacional" ("Responsabilidad del crítico"153).

En concordancia con este paradigma crítico de poesía que se va consolidando en la revista $-\mathrm{y}$ en estrecha sintonía con estas afirmaciones de Portogalo-, en el discurso de Pedroni que Cuadernos publica en su número 14, el poeta santafesino delimitaba los principios de su propia "poiesis" y declaraba: "no he vivido de espaldas a mi pueblo, sino con él y en su drama. [...] y de este 
permanente enlace de lo individual con lo colectivo, he llegado a producir, según vosotros, una obra de contenido humano y social donde el pueblo se encuentra a sí mismo [...]" ("Discurso en Esperanza" 93). Herencia cultural, expresión popular, fidelidad a la clase, enlace entre el poeta y el pueblo, atención a sus dramas son, ciertamente, los tópicos críticos más extendidos que trazan los recorridos de la lectura crítica comunista en torno a la poesía, tal como se ejercita en las páginas de la revista.

Si en el caso de la narrativa, el problema del "elemento revolucionario" y de la "comunicabilidad" derivó en intensas polémicas en torno al carácter "ejemplar" de los argumentos de las obras -lo que, simplificado al extremo, redujo la discusión al problema del "final feliz" (Cfr. Alle "Un boedismo")-, en el caso de la poesía, la conexión con esos principios del realismo pareció resolverse de un modo diferente. A lo largo de toda la década, solo se registra una discusión sobre poesía en la revista, entre Margarita Aguirre y Portogalo, en torno a la reseña que la primera escribe sobre Poemas con habitantes, en el número 23. Aguirre "deplora" que al poemario le falte "fuerza vital" pues "no basta que el poeta se identifique" con los lectores, sino que "se espera" que "nos asegure: "no sufras, porque ganaremos" (171). Esta polémica, que va en la línea del cuestionamiento a la falta de "optimismo" que dominó las discusiones sobre narrativa, se cierra con la respuesta de Portogalo y podría decirse que se trata de un caso excepcional. Así, las políticas editoriales de la revista en torno a la poesía pusieron el foco en el examen de la capacidad del poeta y del poema para "compenetrarse" -según el término que utiliza Manauta (48)- con su pueblo y su entorno, como rasgo ya en sí mismo suficiente para garantizar -ahora en las palabras de Villanueva- la posibilidad de "alumbrar la conciencia" del "lector común", es decir, para cumplir con ese principio transformador al que debe tender toda obra realista ("Pedroni y el color local" 23).

Generalizando los términos a los que recurre Villanueva para caracterizar las búsquedas poéticas de Pedroni, se puede afirmar que la conformación de ese ámbito de poéticas comunistas en la revista Cuadernos se sustentó en la búsqueda 
por abrir "caminos amplios y muy claros" a la poesía a partir de la "identificación fraternal con nuestras más comunes querencias" y de la "naturalidad expresiva"; "dos virtudes" - asociadas a las dimensiones de forma y contenido de la poesía- que Villanueva destaca en una obra poética que, según sus palabras, "nos gana para mejorarnos”. Así, para Villanueva, los valores positivos de la obra de Pedroni estarían concentrados, a nivel formal, en su asimilación a los modos expresivos del pueblo al que está destinada; a nivel temático, en la capacidad de identificación del poeta con los “dramas" de su entorno; $y$, por último, en lo que respecta a su finalidad instrumental, en su potencial para "mejorar" a ese lector que coincide, según el horizonte de expectativas que se pone en juego en estas lecturas, con el objeto de la representación. Estas "virtudes" que Villanueva descubre en la poesía del santafesino ofician de disparador para la definición de una nueva noción de "color local", como atributo peculiar no sólo de la poesía de Pedroni sino, más ampliamente, como rasgo cualitativo al que la "verdadera poesía" no podría sustraerse en tanto "comporta su realidad sensible y su lenguaje natural" (16).

Estas cualidades poéticas que Villanueva destaca en Pedroni componen un núcleo de fuerza que articula las políticas editoriales de promoción y difusión de poesía en la revista Cuadernos. En la dirección trazada por la argumentación de Villanueva, podría entonces plantearse que este concepto de "color local" se constituye como una suerte de denominador común que permite reunir las poéticas que circulan por las páginas de la revista y encontrar un punto de confluencia para pensar su carácter realista en tanto forma de "compenetración" con los "dramas" del pueblo. En efecto, este concepto propicia la conjugación del "elemento revolucionario" y de la "comunicabilidad" ("realidad sensible" y "lenguaje natural") involucrados en el principio del realismo con el tenor nacional y popular que pautaba el programa literario comunista desde un repertorio temático y formal amplio y susceptible de múltiples y diferentes resoluciones.

Según Villanueva, el "verdadero "color local”, lejos de quedar reducido al pintoresquismo o a la "retórica tradicionalista" que busca lo vernáculo en "lo invariable" del pasado -o, como decía Agosti en la década anterior, a los "versitos 
con guitarras y cintas celestes y blancas"-, es "traslaticio, cambiante y susceptible de renovación en sus matices" e incluye todos aquellos aspectos "típicos" de la realidad "con los que se va asimilando simpáticamente la acción creadora del hombre". El color local se hallaría "en todos los elementos activos, vivos y transformables" del paisaje que rodea al poeta, es decir, comprendería todo aquello que se conjuga "secularmente" en la realidad, desde lo aborigen o lo gauchesco hasta las obras de la ingeniería moderna. La amplitud del concepto de Villanueva permite que funcione, entonces, como concepto crítico central para la caracterización de las prácticas poéticas que difunde la revista, en la medida en que ellas apelan a múltiples recursos temáticos y expresivos con los cuales exploran modos posibles de afinidad con diversos aspectos de la realidad local o nacional. De este modo, permite abarcar, por ejemplo, desde la representación de la vida cotidiana y laboral del hombre de provincia, en el caso de la poesía de Pedroni o Manauta, o de la ciudad de Buenos Aires, en la de Portogalo o Tuñón, hasta el "sentimiento del paisaje" regional, que domina la poesía de Manauta y Ortiz; ${ }^{18}$ desde el rescate memorioso de la Buenos Aires de los años 20, en Tuñón, hasta la narración poética de la colonización gringa que distingue la poesía de Pedroni, la historia de los inmigrantes de los conventillos porteños en Portogalo o la vida y las costumbres del gaucho en Villanueva; desde el canto celebratorio del pueblo o de la tierra argentina que se puede leer en Ortiz, Portogalo y Pedroni hasta la recuperación del tango en Portogalo y Tuñón, del folclore en Villanueva y Manauta, de la gauchesca o del lunfardo en Villanueva y Portogalo; pasando también por la denuncia ante sucesos nacionales de actualidad que conmueven a la prensa partidaria, como la desaparición del médico comunista rosarino, Juan Ingalinella, en el poema de Pedroni que publica la revista en su número 23.

Así entendido, el color local se constituiría, además, como una instancia que posibilita a la poesía -y al poeta-acercarse al pueblo y a sus problemas y, por ende, devenir "universal"; lo que Agosti, en su libro del 45, definía como una “argentinidad que es posesión cósmica de la tierra”. En otras palabras, al interpretar

\footnotetext{
${ }^{18}$ La idea de "sentimiento del paisaje" se toma de Ortiz ("El paisaje").
} 
la "íntima realidad de su país", la poesía entraría en diálogo "con la humanidad, sobre palpitantes problemas comunes de la especie, para transmitirle nuestro pensamiento nacional" (Villanueva "Pedroni" 23). Desde los programas partidarios, entonces, la perspectiva nacional de la poesía de estos autores no colisiona sino que asegura su índole humanista y universal. Si la lucha antiimperialista que signó los programas políticos del partido a escala internacional en los años de la Guerra Fría se tradujo, en términos culturales, en la tarea de defensa y revalorización de las culturas nacionales, estas poéticas comunistas, lejos de estar reñidas con la perspectiva internacionalista del PC, aseguran una conexión productiva con los problemas globales. Así, la tendencia nacional y popular de esas poéticas se articula con la toma de posición ante acontecimientos sociales y políticos de gran impacto internacional: la Guerra de Corea, en Ortiz, Guerrero y Tuñón; los crímenes cometidos por el imperialismo norteamericano, como la condena a muerte del matrimonio Rosenberg, en Pedroni y Tuñón; la intervención norteamericana en Guatemala, en Guerrero y Tuñón; la defensa de la paz, en Guerrero, Portogalo, Ortiz y Tuñón; la Revolución Cubana, en Pedroni y Tuñón.

En la dirección de las hipótesis hasta aquí desarrolladas, es interesante detenerse en la publicación de "Entre dos ríos" de Manauta en el número 27 y, dos números después, del adelanto del poemario A la sombra de los barrios amados, de Tuñón. En los dos casos pueden rastrearse similares decisiones de edición y diagramación, lo que habilita a pensar que la revista intentó llevar adelante el proyecto editorial de publicación de una sección específica de poesía con poemarios breves, adelantos o selecciones de poemas de los autores de relieve dentro de la revista y del espacio cultural comunista que, por razones desconocidas, no se sostuvo en el tiempo. De hecho, en ambos casos los poemarios se disponen hacia el final del número, separados del resto de la revista con una página inicial en la que se consigna el título y en cuya contracara (la carilla par) se añade una ilustración -de Andrés Calabrese, en Entre dos ríos; de Enrique Policastro, en el caso de A la sombra-que enmarca a los poemas y les otorga cierta independencia del resto de la revista: como si se tratara de un pequeño folleto incluido en el interior mismo del 
número. El hecho de que la selección de Tuñón se inicie con una nota que oficia de prólogo marca una diferencia respecto de la publicación anterior del poemario de Manauta. Por otro lado, en el número 27 se publica también un extenso artículo de Roberto Salama, "El realismo de Manauta", sobre la novela del autor recientemente publicada, Las tierras blancas, que de algún modo tiende un lazo de diálogo con los poemas que se ubican hacia el final de ese mismo número. Ahora bien, la lectura en filigrana de ambas publicaciones viene a corroborar varias ideas. En primer lugar, la selección de poemas de un poeta entrerriano y de otro como Tuñón, identificado desde los años 20 con la ciudad de Buenos Aires y sus márgenes cosmopolitas y populares, ilumina esa "política poética" de la revista y de los programas culturales del PC sustentada en la construcción de una tradición poética nacional capaz de extender sus alcances desde el centro del campo literario hacia las escalas provinciales y locales. En segundo lugar, la decisión editorial de incluir los poemas de ambos poetas, tan distantes a nivel expresivo como temático, permite ilustrar ese concepto de "color local" definido por Villanueva como núcleo de fuerza que articula las estrategias de promoción y difusión de poesía en la revista Cuadernos ligada a una búsqueda de lo nacional sin quedar atrapada en las fauces del regionalismo, el nativismo o el criollismo. En efecto, si la poesía de Manauta, desde un registro simbolista, constituye una “'descripción poética' de la provincia, desplegada a partir de la geografía, donde el río y la luz son primordiales" (Galeano 32), la de Tuñón, en cambio, involucra "una voluntad de recuperación de Buenos Aires como ciudad entrañable y como arrabal del mundo" (Viñas 169). Desde el centro porteño hasta el cauce de los ríos entrerrianos, desde el recuerdo de una Buenos Aires ya ausente que se adivina todavía en algunos rincones de la ciudad actual hasta la vida esforzada, laboriosa y siempre acorralada por el hambre de los hombres de campo, la poesía comunista se extiende geográficamente y amplifica su repertorio temático en vistas abarcar todos aquellos aspectos "típicos" de la realidad "con los que se va asimilando simpáticamente la acción creadora del hombre". En última instancia, la elección de poemas de Manauta y Tuñón para integrar esos números de Cuadernos echa luz sobre el interés de la revista por 
conformar un ámbito específico de poéticas comunistas capaces de explorar las posibilidades de la poesía para "compenetrarse" con el pueblo y "alumbrar" su conciencia. Por último, es necesario destacar la inclusión específica de ese poemario de Tuñón, que da inicio a una nueva -y última- etapa de su producción poética, en la que la poesía se convierte en un vasto espacio de resguardo del pasado, que se sustancia en la recuperación poética de objetos en desuso, anécdotas de la juventud, historias de pérdida y violencia, lugares que el tiempo ha modificado y nombres propios: desde los muertos que dejaron las guerras que presenció y las víctimas de la injusticia, hasta las antiguas victrolas o las fotos corroídas; desde el cine mudo, la casa infantil o la Buenos Aires de los años 20 con sus fondines y calesitas, hasta la tradición literaria universal; desde los viejos organitos o los tangos de ayer hasta las luchas por las reivindicaciones sociales y políticas de las que participó. A la luz del programa poético del PCA que se define desde Cuadernos es posible leer ese "resguardo del pasado" en su poesía, esa voluntad de reconstrucción de la Buenos Aires de los años 20, en relación a la búsqueda por recuperar lo "típico" de la realidad nacional. En esta dirección, como decía más arriba, si por un lado el interés por armar una tradición cultural nacional reorganiza el campo de la poesía al punto de otorgar centralidad a los poetas de provincias, por otro, conlleva una reorientación en las búsquedas poéticas de los poetas porteños, ahora ligadas a explorar los matices ciudadanos del "color local".

\section{De las poéticas comunistas de los años 50 hacia las de los 60}

En la nota titulada "Un debate sobre poesía" que se publica en el número 49 de Cuadernos (de septiembre-octubre de 1960), un encubierto "Espectador" transcribe las discusiones que tuvieron lugar en la reunión de "poetas comunistas" convocada el 16 de julio de ese año por la Comisión de Asuntos Culturales del PC. Si bien se aclara en la nota introductoria que la reunión fue "necesariamente restringida" por las circunstancias sociales y políticas y que participaron poetas de 
capital y del interior del país, interesa señalar que, salvo Tuñón-además de Héctor Agosti que presidió el encuentro, aunque no era poeta-, el resto de los poetas cuyas declaraciones se reproducen en la nota pertenecen a una nueva promoción de “poetas comunistas", entre los que se encuentran Juan Gelman, Luis Navalesi, Juana Bignozzi, Rosario Mase, Héctor Negro, Julio Huasi -integrantes todos ellos del colectivo de poesía "El pan duro"- y otros como Carlos Alberto Brocato, quien será a partir de 1964 uno de los directores de la revista La rosa blindada (19641966). ${ }^{19}$ Como señalé al comienzo de este trabajo, estos jóvenes poetas comienzan a hacer sus primeras incursiones en la crítica literaria y cultural hacia mediados de la década del 50 en la revista Cuadernos; espacio en el que, además, se difunden sus primeros libros y tienen lugar las principales polémicas y discusiones que van a ir señalando el camino hacia el definitivo alejamiento, unos años después, de las estructuras partidarias, con la conformación de un programa en abierta ruptura con los lineamientos hegemónicos del partido y el montaje de sus propios medios de difusión cultural. Según la nota (y descontando a Tuñón, quien sin dudas era el poeta comunista argentino de mayor relevancia desde varias décadas atrás), no participaron de ese debate $-\mathrm{O}$, al menos, no se tuvieron en cuenta sus exposiciones para su difusión- los otros poetas que, hasta ese momento, eran los principales dinamizadores de la revista Cuadernos y aquellos que más difusión tenían en sus páginas.

Pero, además, los nombres de los poetas participantes de este debate parece mostrar que la comisión de cultura del partido otorgaba cierta importancia a las

${ }^{19}$ Los jóvenes del grupo de poesía "El pan duro" rondaban los 20 años cuando comenzaron a reunirse, en 1955, año clave para la vida política argentina, tal como ellos mismos lo explican en el prólogo a la antología colectiva que, en 1963, publican bajo el sello editorial La rosa blindada. Las principales actividades del grupo en sus comienzos estuvieron abocadas, por un lado, a la realización de lecturas públicas de poemas en teatros, bares, bibliotecas, sindicatos y clubes; y, por otro, a la publicación de sus poemarios mediante un sistema de venta de vales anticipados con los cuales costeaban los gastos de edición. La rosa blindada, por su parte, fue un importante emprendimiento editorial de un grupo de jóvenes de izquierda que, hacia 1964, comenzó a publicar una revista con el mismo nombre. Dirigida por Carlos Brocato y José Luis Mangieri y con la activa colaboración Andrés Rivera, Carlos Gorriarena, Norberto Onofrio y de varios de los integrantes del colectivo de poesía "El pan duro", entre ellos, Juan Gelman y Guillermo Harispe, la revista contó con nueve números, el último de ellos publicado en 1966. 
opiniones de estos jóvenes, aunque también podría ser un indicador de la necesidad de acordar con ellos, ante ciertas disidencias que ya comenzaban a despuntar, algunos aspectos básicos de cualquier creación poética que se precie de "comunista". De hecho, las intervenciones en ese debate de Gelman, Huasi, Bignozzi o Mase, por ejemplo, iluminan los puntos conflictivos con las perspectivas que prioriza la agenda cultural del PC, al plantear la necesidad de apertura hacia una reconsideración de la importancia de la experimentación a nivel formal, $\mathrm{y}$, de manera concomitante, hacia una relectura de las vanguardias o, más ampliamente, de las experiencias de la modernidad literaria. Distante de esa "comunicabilidad" que pautaban los programas poéticos comunistas, los jóvenes poetas están comenzando a alentar, en términos de Bignozzi, una vanguardia que se ejerza "no sólo en el plano político sino también en el plano artístico" (Espectador 116) contra lo que Gelman denomina las “formas tiránicas de la unidad de expresión".

Las relaciones de encuentros y desencuentros que Cuadernos mantiene con la revista Poesía Buenos Aires, una de las expresiones de la renovación poética más importante de la década, ilustran muy bien las dificultades que el programa poético de la revista tiene para comprender y validar cualquier camino de exploración formal que aleje al poema de esa "comunicabilidad" que plantea como imprescindible para la creación poética popular. En efecto, por un lado, en Cuadernos se destaca la antología que el grupo Poesía Buenos Aires publica en 1954, Guatemala, por el interés que la misma evidencia por "sumarse a la condena por la agresión pirata" (Hidalgo 121), y, en las conclusiones del "Debate", Agosti reconoce la "incipiente toma de conciencia" (119) del grupo. Pero, por otro, se critica su "defensa de la forma" por sobre el contenido (Hidalgo 121) o el recurso excesivo a "lo indirecto", "lo sugerente", como sostiene Brocato en el debate sobre poesía (Espectador 108)-. ${ }^{20}$ En esta dirección, podría afirmarse que la política

\footnotetext{
${ }^{20}$ Al mismo tiempo, si como señala Martín Prieto ("En el aura del sauce”), Poesía Buenos Aires publica poemas de Ortiz en 1955, lo que indicaría un interés de filiación, es posible pensar que la figura del poeta entrerriano se convierte en un objeto compartido, y en disputa, entre ambos grupos. En esta dirección, en su reseña de El alma y las colinas, Manauta alerta a los "estetas" a
} 
poética de Cuadernos es refractaria a la experimentación formal, más allá de que el contenido que se exprese sí sea acorde con los mismos postulados del comunismo. Puestos en la balanza, contenido revolucionario y forma clara, "comunicable", deben mantenerse en estricto equilibrio. Lo que los jóvenes poetas comunistas están comenzando a plantear es, en términos de Bignozzi, que "no podemos juzgar, como en alguna medida se lo hace, cierto tipo de arte por el hecho de que sea entendido o no" (Espectador 116).

Más allá de esta conjetura acerca del posible interés partidario por “encauzar" las prácticas creativas de estos jóvenes, lo cierto es que el hecho de que ese debate haya privilegiado sus intervenciones evidencia un claro desvío en los lineamientos del programa poético comunista de los 50; un desvío que no implicaría un desplazamiento sino más bien una coexistencia de dos promociones poéticas, tal como permite inferir que en ese mismo número de la revista se publique "Poesía para el hombre", el discurso que Pedroni lee en ocasión de recibir el premio “Alberto Gerchunoff” por Cantos del hombre. Pero, además, hay que señalar que esa coexistencia no se extenderá en el tiempo: cuatro años después, en un contexto signado por la relectura del peronismo y el impacto de la Revolución Cubana-ejes de una discusión que involucra un posicionamiento ante muchos otros acontecimientos sociopolíticos contemporáneos-, estos poetas de la "nueva izquierda" serán expulsados del partido por sus disidencias con las líneas culturales y políticas dogmáticas, provocando una fractura sin retorno en el sector de la izquierda comunista del campo intelectual y literario.

no "engañarse" pues "las alturas" de la poesía de Ortiz "arraigan en la tierra" ("Juan L. Ortiz" 142). Si bien no especifica nombres ni grupos, puede leerse allí una impugnación de otras lecturas de la obra de Ortiz y un intento por reapropiarla para el programa poético comunista. 


\section{Observaciones finales}

A lo largo de este trabajo he intentado dar cuenta de la conformación de un ámbito de poéticas comunistas en la revista Cuadernos de Cultura, en el marco de la recepción de la doctrina del zhdanovismo cultural en la Argentina de la década de 1950. En primer lugar, la descripción de las notas, artículos críticos y reseñas de poesía que se publican en sus páginas en el transcurso de esa década me permitió mostrar la importancia que adquiere la reflexión en torno a la poesía dentro del espectro del interés central que constituye la literatura. Al mismo tiempo, la recurrencia de los nombres de ciertos poetas argentinos provenientes tanto del centro metropolitano como de las provincias del interior del país, como activos participantes y como objeto de interés -Pedroni, Manauta, Villanueva, Portogalo, Guerrero, Ortiz, Tuñón- posibilitó la identificación de un corpus original de poéticas comunistas que hasta el momento no ha sido estudiado por la crítica especializada. En efecto, el trabajo de investigación realizado en este artículo vincula a un grupo de poetas cuyas producciones, hasta ahora, no habían sido puestas en diálogo bajo el paraguas de los programas realistas que sostiene el PCA en los años 50 en vistas de la construcción de una tradición poética nacional, vinculada a los problemas del pueblo. Desde este punto de vista, se examinó la reconfiguración del campo literario que propicia la revista Cuadernos de Cultura en función de la conformación de un ámbito específico de poéticas comunistas identificadas por su tendencia nacional, que favorecerán una reorientación en los parámetros valorativos en torno de los cuales se lee, se critica y se proyecta la escritura de poesía.

En segundo lugar, entonces, avancé en el análisis de los términos críticos que permiten agrupar, bajo el paraguas del realismo, producciones poéticas como las de los poetas del corpus, cuyas búsquedas expresivas y temáticas son diversas. Con el propósito de lograr la "confluencia de todas las voces provincianas en un registro común”, según vimos en la cita de Larra, los análisis de poesía que propicia la revista centran su interés en los diversos modos de acercamiento a temáticas, 
lenguajes, expresiones culturales "nacionales", de raigambre popular, pero alejados de las simplificaciones del costumbrismo, del nativismo o del criollismo que Agosti criticaba en la década anterior. En esta dirección, hallé en el concepto de "color local" que elabora Villanueva en su artículo sobre la poesía de Pedroni, un conjunto de ideas acerca de la creación poética que permite la articulación de las disímiles producciones de los autores que circulan por las páginas de la revista en el marco de la búsqueda por construir una tradición poética nacional, ligada al pueblo. El "color local", para Villanueva, supone a nivel formal la asimilación a los modos expresivos del pueblo al que está destinada y, a nivel temático, la capacidad de identificación del poeta con los "dramas" de su entorno, desde una amplia gama de registros y resoluciones; lo que permitiría el cumplimiento de aquella finalidad instrumental que pautaba el programa realista de contribuir a la transformación de la realidad. El "color local", tal como lo define Villanueva, aparece así desvinculado del regionalismo y ofrece la amplitud temática y expresiva necesaria para reunir a los poetas estudiados.

Por último, consideré el debate sobre poesía que organiza el PC en 1960 y luego se publica en la revista para pensar las tensiones entre las poéticas comunistas de los años 50 y aquellas otras que, en la década siguiente, renovarán las búsquedas artísticas de la poesía a partir del cuestionamiento a lo que definen como "uniformidad expresiva", en vistas fundamentalmente de repensar la importancia de la experimentación vanguardista. No obstante, como vimos, ese interés de renovación que plantean los jóvenes poetas de los 60 los lleva, apenas unos años después, a desvincularse del partido. 


\section{Bibliografía}

\section{Corpus}

Cuadernos de Cultura (Primera Época), $\mathrm{n}^{\circ} 1$ (agosto 1950); $\mathrm{n}^{\circ} 84$ (marzo-abril 1967).

AA.VV. El Pan Duro. Grupo de poesía. Buenos Aires: La Rosa Blindada, 1963. Agosti, Héctor. Defensa del realismo. Montevideo: Ediciones Pueblo Unido, 1945.

Aguirre, Margarita. "Responsabilidad del poeta". Cuadernos de Cultura 23 (1955): 170-171.

Espectador. "Un debate sobre poesía”. Cuadernos de Cultura 49 (1960): 101-120. Gorki, Máximo. "Discurso en el Primer Congreso de Escritores Soviéticos (1934)”. Máximo Gorki y Andréi Zhdanov. Literatura, filosofía y marxismo. México D.F.: Grijalbo, 1968.

Hidalgo, Juan. "Guatemala”. Cuadernos de Cultura 21 (1955): 121-123.

Larra, Raúl. "Gerardo Pisarello: Pan curuica”. Cuadernos de Cultura 29 (1957): 108-110.

Manauta, Juan José. “'Canto General': culminación del tema del hombre en la poesía de Pablo Neruda”. Cuadernos de Cultura 8 (1952): 40-49. . "Juan L. Ortiz. 'El alma y las colinas"”. Cuadernos de Cultura 28 (1957): 142.

Ortiz, Juan L. "El paisaje en los últimos poetas entrerrianos". Obra completa. Santa Fe: UNL Ed, 205.

Pedroni, José. "Discurso en Esperanza”. Cuadernos de Cultura 14 (1954): 91-97.

Portogalo, José. "Responsabilidad del crítico". Cuadernos de Cultura 24 (1956): 151-154.

Villanueva, Amaro. "Pedroni y el color local". Cuadernos de Cultura 17 (1954): $12-23$.

Yunque, Álvaro. Poetas sociales de la Argentina. Tomo I y II. Buenos Aires: Problemas, 1943. 
CATEDRAL Tomada: Revista literaria latinoamericana / Journal of Latin American Literary Criticism María Fernanda Alle

Zhdanov, Andréi. "El frente ideológico y la literatura". Máximo Gorki y Andréi Zhdanov. Literatura, filosofia y marxismo. México D.F.: Grijalbo, 1968.

\section{Bibliografía crítica e historiográfica citada}

Alle, María Fernanda. "Un boedismo optimista. El realismo socialista en Argentina a la luz de un concurso de cuentos de la revista Cuadernos de Cultura". Izquierdas n 37 (2017): 11-32.

Alzari, Agustín. “La poesía social de Juan L. Ortiz (1936-1946)”. Tesis Doctoral, Facultad de Humanidades y Ciencias de la Educación, Universidad Nacional de La Plata. Memoria académica, 2016. Web. 12 diciembre 2018.

Bourdieu, Pierre. Las reglas del arte. Génesis y estructura del campo literario. Barcelona: Anagrama, 1995.

Cernadas, Jorge. "La 'vieja izquierda' en la encrucijada: Cuadernos de Cultura y la política cultural del Partido Comunista argentino (1955-1963)". X Jornadas Interescuelas / Departamentos de Historia. Escuela de Historia, Facultad de Humanidades y Artes, UNR; Departamento de Historia, Facultad de Ciencias de la Educación, UNL, Rosario. Acta académica, 2005. Web. 12 diciembre 2018.

Crespo, Horacio. "Poética, política, ruptura". La irrupción de la crítica, vol. X. Susana Cella, directora del volumen. Historia crítica de la literatura argentina. Noé Jitrik, director general. Buenos Aires: Emecé, 1999.

Galeano, Gabriela. "Introducción”. Juan José Manauta, Poesía completa. Paraná: Eduner, 2015.

Lecourt, Dominique. Ensayo sobre la posición de Lenin en la filosofía. México D.F.: Siglo XXI, 1979.

Longoni, Ana y Horacio Tarcus. "Purga antivanguardista. Crónica de la expulsión de Córdova Iturburu del Partido Comunista". Ramona 14 (2001): 55-57. Web. 12 diciembre 2018. 
Martínez, Ana Teresa. (2013). "Intelectuales de provincia: entre lo local y lo periférico". Prismas vol. 17 n 2 (2013): 169-180.

Petra, Adriana. Intelectuales comunistas en la Argentina (1945-1963). Tesis de posgrado, Universidad Nacional de La Plata, Facultad de Humanidades y Ciencias de la Educación. Memoria Académica, 2013. Web. 12 diciembre 2018. . Intelectuales y cultura comunista. Itinerarios, problemas y debates en la Argentina de posguerra, Buenos Aires: Fondo de Cultura Económica, 2018 .

Prieto, Martín. "En el aura del sauce en el centro de una historia de la poesía argentina”. Juan L. Ortiz. Obra Completa. Santa Fe: UNL Ed., 2005.

Sánchez Vázquez, Adolfo. “El concepto de praxis en Lenin”. Diánoia n 25 (1979): 46-61. Web. 14 de febrero de 2019.

Sapiro, Gisèle. "Le champ est-il national? La théorie de la différenciation sociale au prisme de l'histoire globale". Actes de la recherche en sciences sociales $\mathrm{n}^{\circ} 200$ (2013): 70-85.

Slonim, Marc. Escritores y problemas de la literatura soviética. 1917-1967. Madrid: Alianza Editorial, [1964] 1974.

Tarcus, Horacio. "El corpus marxista". La irrupción de la crítica. Susana Cella, directora del volumen. vol X. Historia crítica de la literatura argentina. Noé Jitrik, director general. Buenos Aires, Emecé, 1999.

Viñas, David. “Cinco entredichos con Raúl González Tuñón”. Literatura argentina y politica. De Lugones a Walsh. Buenos Aires: Sudamericana, 1996.

Volkov, Solomon. El coro mágico. Una historia de la cultura rusa de Tolstói a Solzhenitsyn. Barcelona: Ariel, 2010. 